\title{
A fuzzy adaptive sliding mode controller for uncertain nonlinear multi motor systems
}

\author{
Tran Xuan Tinh ${ }^{1}$, Pham Tuan Thanh ${ }^{1}$, Tran Van Tuyen ${ }^{1}$, Nguyen Van Tien ${ }^{1}$ and Dao Phuong Nam ${ }^{2, *}$ \\ ${ }^{1}$ Le Quy Don University (Military Technical Academy), Vietnam \\ ${ }^{2}$ School of Electrical Engineering, Hanoi University of Science and Technology, Vietnam
}

\begin{abstract}
Multi-motor drive systems are nonlinear, multi-input multi-output (MIMO) and strong-coupling complicated system, including the effect of friction and elastic, backlash. They have been widely used in many modern industries. The control law for this dive system much depend on the determining of the tension being hard to obtain this tension in practice based on a load cell or a pressure meter due to the accuracy of sensors or external disturbance. An emerging proposed technique in the control law is the use of adaptive sliding mode control scheme to stabilize closed system. However, the control system would be affected by chattering phenomenon. In order to eliminate this term, fuzzy technique is proposed by adjusting equivalent coefficients. The theory analysis and simulation results point out the good performance of the proposed fuzzy adaptive sliding mode control for the drive system.
\end{abstract}

\section{Introduction}

Multi-motor drive systems have been investigated by many researchers in the recent times. The neural network technique based control law have been proposed by Yaoji Me et al. (2013) (see [1-9]). However, it is hard to find the equivalent networks as well as corresponding learning rules. Besides, the model of this system is approximately described as a linear system to use the transfer function to design the control law. Furthermore, the tracking ability or the stabilization of the whole system are not still solved under the effects of neural network based observer. In the multi-motor drive control systems, it is necessary to obtain the belt tension to design the suitable state feedback control law. However, it is hard to measure this belt tension based on sensors, ... and the high gain technique based observer is proposed in our work. Besides, the state feedback control design based on sliding mode control technique ensure that it is easy to remove efficient of disturbance and uncertainties. Therefore, an adaptive sliding mode controller is proposed to obtain tracking effectiveness. Moreover, fuzzy technique is considered to eliminate the chattering phenomenon disappearing by sliding mode control. The stability of closed system is obtained and verified by theory analysis, simulations.

\section{Problem statements}

Due to the effects by backlash and elastic (Fig. 1) and parameters (Table 1), we extend the model in [1] to obtain the following dynamic equation $(2,3)$ and the corresponding transfer function diagram (Fig. 2):

$$
\left\{\begin{array}{l}
\dot{\omega}_{r 1}=\frac{1}{J_{L 1}}\left[c_{1} \cdot f_{11}\left(\Delta \varphi_{1}\right)+b_{1} \Delta \omega_{1} f_{12}\left(\Delta \varphi_{1}\right)-\left(\mathrm{T}_{L 1}+\mathrm{r}_{1} \mathrm{~F}\right)\right] \\
\dot{\omega}_{r 2}=\frac{1}{J_{L 2}}\left[c_{2} \cdot f_{21}\left(\Delta \varphi_{2}\right)+b_{2} \cdot \Delta \omega_{2} f_{22}\left(\Delta \varphi_{2}\right)-\left(\mathrm{T}_{L 2}+\mathrm{r}_{2} \mathrm{~F}\right)\right] . \\
\dot{F}=C_{12}\left[r_{1} \omega_{r 1}-r_{2} \omega_{r 2}\left(1+\frac{1}{C_{12} . l} \mathrm{~F}\right)\right]
\end{array}\right.
$$

Table 1. Parameters of a Multi-Motor System.

\begin{tabular}{|c|c|}
\hline$K=E / V$ & Transfer function \\
\hline$E$ & Young's Modulus of belt \\
\hline$V$ & Expected line velocity \\
\hline$T=\frac{L_{0}}{A V}$ & Time constant of tension variation \\
\hline$L_{0}, A$ & $\begin{array}{l}\text { Distance between racks, Section area } \\
\left(\mathrm{m}^{2}\right)\end{array}$ \\
\hline$n_{p i}$ & Number of pole-pairs in the $\mathrm{i}^{\text {th }}$ Motor \\
\hline $\begin{array}{c}\mathrm{J}_{1}, \mathrm{~J}_{2} \\
\mathrm{~J}_{\mathrm{L} 1}, \mathrm{~J}_{\mathrm{L} 2}\end{array}$ & $\begin{array}{l}\text { Inertia moment of Motors and Loads } \\
\left(\mathrm{kgm}^{2}\right)\end{array}$ \\
\hline $\begin{array}{c}T, \mathrm{~T}_{L} \\
\varphi_{r}\end{array}$ & $\begin{array}{l}\text { Motor, Load torque }(\mathrm{Nm}), \text { Flux of } \\
\text { rotor }(\mathrm{Wb})\end{array}$ \\
\hline$L_{r}$ & Self-induction of rotor $(\mathrm{H})$ \\
\hline $\begin{array}{l}\mathrm{c}_{1}, \mathrm{c}_{2} \\
\mathrm{~b}_{1}, \mathrm{~b}_{2}\end{array}$ & Stiffness and friction coefficient \\
\hline$\Delta \omega_{1}, \Delta \omega_{2}$ & $\begin{array}{l}\text { The errors of angle speed } \\
\text { in presence of backlash, elastic }\end{array}$ \\
\hline
\end{tabular}

We denote:

\footnotetext{
Corresponding author: nam.daophuong@hust.edu.vn
} 


$$
\begin{aligned}
& u_{d 1}=\frac{1}{J_{L 1}}\left[c_{1} f_{11}\left(\Delta \varphi_{1}\right)+b_{1} \Delta \omega_{1} f_{12}\left(\Delta \varphi_{1}\right)\right] \\
& u_{d 2}=\frac{1}{J_{L 2}}\left[c_{2} f_{21}\left(\Delta \varphi_{2}\right)+b_{2} \Delta \omega_{2} f_{22}\left(\Delta \varphi_{2}\right)\right]
\end{aligned}
$$

are components including backlash and elastic to obtain the following equations:

$$
\left\{\begin{array}{c}
\dot{\omega}_{r 1}=-c_{1} \omega_{r 1}-\frac{1}{J_{L 1}} \mathrm{~T}_{L 1}-\frac{1}{J_{L 1}} \mathrm{r}_{1} \mathrm{~F}+u_{d 1} \\
\dot{\omega}_{r 2}=-c_{2} \omega_{r 2}-\frac{1}{J_{L 2}} \mathrm{~T}_{L 2}-\frac{1}{J_{L 2}} \mathrm{r}_{2} \mathrm{~F}+u_{d 2} . \\
\dot{F}=C_{12} r_{1} \omega_{r 1}-C_{12} r_{2} \omega_{r 2}-\frac{1}{l} \omega_{r 2} \mathrm{~F}
\end{array}\right.
$$

The model is described by eq. (3) belongs to the class of nonlinear systems as follows:

$$
\left\{\begin{array}{l}
\dot{x}(\mathrm{t})=A(\mathrm{x}, \mathrm{t}) x+B(\mathrm{x}, \mathrm{t}) u+D(\mathrm{x}, \mathrm{t}) \\
\mathrm{y}=x
\end{array},\right.
$$

where

$$
\begin{aligned}
& A=\left[\begin{array}{ccc}
k_{1} & 0 & k_{2} \\
0 & k_{4} & k_{5} \\
0 & 0 & k_{7} x_{2}
\end{array}\right] ; B=\left[\begin{array}{ccc}
k_{3} & 0 & 0 \\
0 & k_{6} & 0 \\
0 & 0 & k_{8} x_{1}+k_{9} x_{2}
\end{array}\right] \text {; } \\
& D=\left[\begin{array}{c}
u_{d 1} \\
u_{d 2} \\
0
\end{array}\right]
\end{aligned}
$$

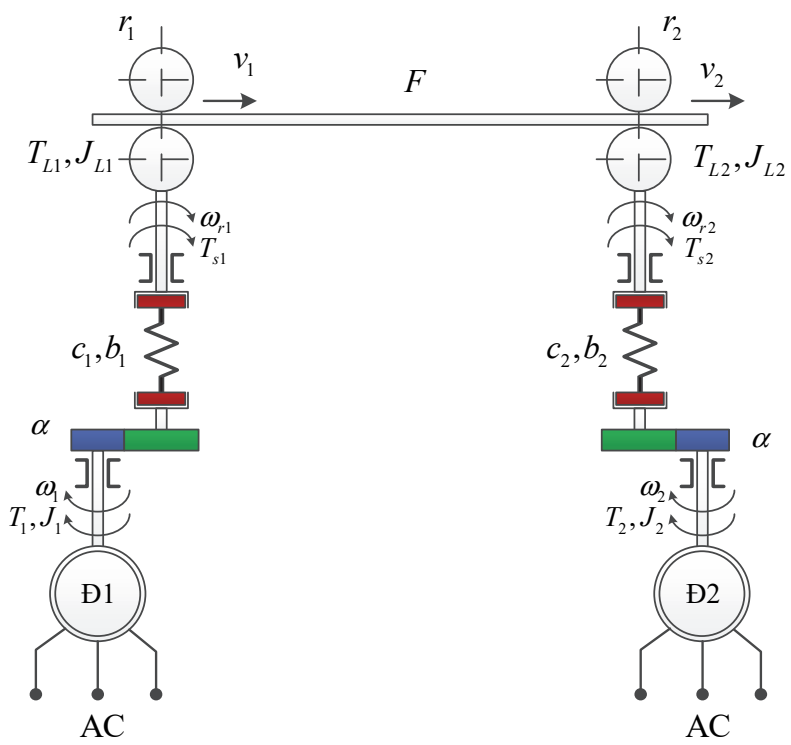

Fig. 1. The Two-Motor Drive System.

\section{Remark 1:}

The dynamic equations $(1,2,3)$ and Figures 1, 2 are described by the effect of friction, backlash, elastic and pointed out the nonlinear property of multi-motor systems.

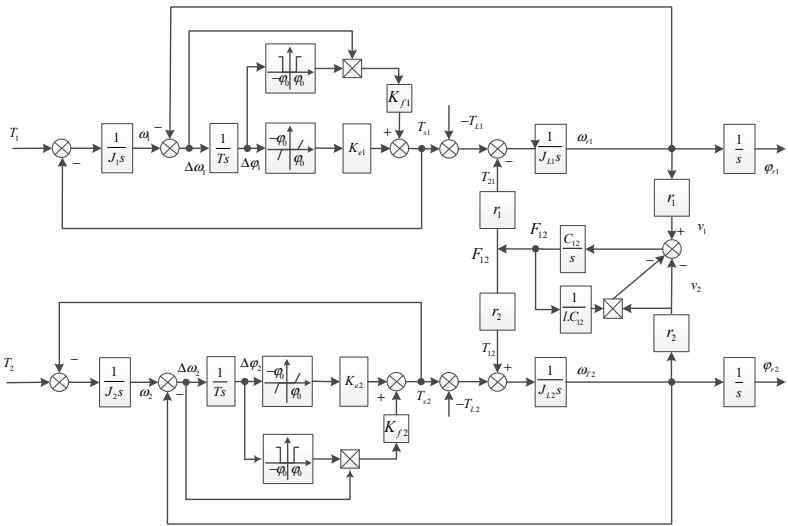

Fig. 2. The corresponding transfer-function diagram of the TwoMotor Drive System.

The control objective is to find the control input vector $u^{T}=\left[\begin{array}{lll}\mathrm{T}_{L 1} & \mathrm{~T}_{L 2} & C_{12}\end{array}\right]$ to obtain that the desired value are tracked by $x^{T}=\left[\begin{array}{lll}\omega_{r 1} & \omega_{r 2} & F\end{array}\right]$ in presence of friction and elastic.

The following assumptions must be satisfied in order to design the control law:

Assumption 1: $x^{T}=\left[\begin{array}{lll}\omega_{r 1} & \omega_{r 2} & F\end{array}\right]$ is measurable;

Assumption 2: There are real positive numbers $\omega_{r 1 \text { max }}, \omega_{r 2 \max }, T_{L 1 \text { max }}, T_{L 2 \text { max }}, u_{d 1 \text { max }}, u_{d 2 \text { max }}$ such that $\omega_{r 1}, \omega_{r 2}, T_{L 1}, T_{L 2}, u_{d 1}, u_{d 2}$ are bounded by these values;

\section{Fuzzy adaptive sliding mode control design}

In this section, the main work is to find a state feedback control law based on the adaptive sliding mode control technique for the class of multi motor systems.

The proposed control law based on the following theorems as follows:

Theorem 1: The adjusting mechanism (5):

$$
\left\{\begin{array}{l}
\dot{\theta}_{a}^{t}=\eta_{a} \alpha \psi_{a}(\mathrm{x}) \mathrm{s}, \\
\dot{\theta}_{b}^{t}=\eta_{b} \alpha \psi_{b}(\mathrm{x}) \mathrm{su}_{e q},
\end{array}\right.
$$

with $s=\alpha e+\xi$,

$$
\begin{aligned}
& u_{e q}(\mathrm{t})=\hat{B}^{-1}(\mathrm{x})\left(-\hat{A}(\mathrm{x})+\dot{x}_{d}-\beta \operatorname{sgn}(\mathrm{s})\right)= \\
& u_{e q}=\hat{B}^{T}\left(\mathrm{x}, \theta_{b}^{t}\right)\left[\varepsilon_{0} I_{m}+\hat{B}\left(\mathrm{x}, \theta_{b}^{t}\right) \hat{B}^{T}\left(\mathrm{x}, \theta_{b}^{t}\right)\right]^{-1} ; \\
& \left(-\hat{A}\left(\mathrm{x}, \theta_{a}^{t}\right)+\dot{\mathrm{x}}_{d}-\beta \operatorname{sgn}(\mathrm{s})\right) \\
& A(x) \approx \hat{A}\left(x, \theta_{a}^{t}\right)=\theta_{a} \psi_{a}(\mathrm{x})=\sum_{i=1}^{p_{a}} \theta_{a i} \psi_{a i}(\mathrm{x}) ; \\
& \mathrm{B}(x) \approx \hat{\mathrm{B}}\left(x, \theta_{b}^{t}\right)=\theta_{b} \psi_{b}(\mathrm{x})=\sum_{i=1}^{p_{b}} \theta_{b i} \psi_{b i}(\mathrm{x}) .
\end{aligned}
$$

ensure some results as follows:

1. All signals of closed loop will be bounded and $\theta_{a}, \theta_{b}$ converge to 


$$
\begin{aligned}
& \theta_{a}^{*}=\underset{\theta_{a i}}{\arg \min }\left\{\sup _{x \in \Omega}\left|A(x, t)+D(x, t)-\hat{A}\left(x, \theta_{a i}\right)\right|\right\} \\
& \theta_{b}^{*}=\underset{\theta_{b i}}{\arg \min }\left\{\sup _{x \in \Omega}\left|B(x, t)-\hat{A}\left(x, \theta_{a i}\right)\right|\right\}
\end{aligned}
$$

as $t \rightarrow \infty$

2. If $A^{*}\left(x, \theta_{a}^{*}\right)=\hat{\mathrm{A}}\left(x, \theta_{a}^{t}\right) ; \mathrm{B}^{*}\left(x, \theta_{b}^{*}\right)=\hat{B}\left(x, \theta_{b}^{t}\right)$ then the errors converge to zero in finite time;

3. If $A^{*}\left(x, \theta_{a}^{*}\right) \neq \hat{\mathrm{A}}\left(x, \theta_{a}^{t}\right) ; \mathrm{B}^{*}\left(x, \theta_{b}^{*}\right) \neq \hat{B}\left(x, \theta_{b}^{t}\right)$ then closed loop system converges to the neighborhood of sliding surface in finite time;

\section{Proof:}

The Lyapunov candidate function is selected as follows:

$$
V=\frac{1}{2} s^{2}+\frac{1}{2} \frac{1}{\eta_{a}} \tilde{\theta}_{a}^{2}+\frac{1}{2} \frac{1}{\eta_{b}} \tilde{\theta}_{b}^{2}
$$

where $\tilde{\theta}_{a}=\theta_{a}-\theta^{*} ; \tilde{\theta}_{b}=\theta_{b}-\theta_{b}^{*}$, we have:

$$
\dot{V}=s \dot{s}+\tilde{\theta}_{a} \dot{\tilde{\theta}}_{a}+\tilde{\theta}_{b} \dot{\tilde{\theta}}_{b}
$$

and:

$$
\begin{gathered}
\dot{s}=\alpha \dot{e}=\alpha\left(\dot{x}-\dot{x}_{d}\right) \\
=\alpha\left(\begin{array}{l}
A(x, t)+B(x, t) u(t)+D(x, t) \\
-\hat{A}(x, t)-\hat{B}(x, t) u_{e q}(t)-\beta \operatorname{sgn}(\mathrm{s})
\end{array}\right) \\
=\alpha\left(\begin{array}{l}
(A(x, t)+D(x, t)-\hat{A}(x, t)) \\
+(B(x, t)-\hat{B}(x, t)) u_{e q}(t)-B(x, t) u_{c}(t)-\beta \operatorname{sgn}(\mathrm{s})
\end{array}\right)
\end{gathered}
$$

However, we have the relation:

$$
\begin{aligned}
& \varepsilon_{a}(x, t)+A^{*}\left(x, \theta_{a}^{*}\right)-\hat{A}\left(x, \theta_{a}\right)=A(x, t)+D(t)-\hat{A}\left(x, \theta_{a}\right) \\
& \varepsilon_{b}(x, t)+B^{*}\left(x, \theta_{b}^{*}\right)-\hat{B}\left(x, \theta_{b}\right)=B(x, t)-\hat{B}\left(x, \theta_{b}\right)
\end{aligned}
$$

Therefore, we obtain:

$$
\dot{s}=\alpha\left[\begin{array}{l}
\left(\varepsilon_{a}(x, t)+A^{*}\left(x, \theta_{a}^{*}\right)-\hat{A}\left(x, \theta_{a}\right)\right) \\
+\left(\varepsilon_{b}(x, t)+B^{*}\left(x, \theta_{b}^{*}\right)-\hat{B}\left(x, \theta_{b}\right)\right) u_{e q}(t) \\
-B(x, t) u_{c}(t)-\beta \operatorname{sgn}(\mathrm{s})
\end{array}\right]
$$

On the other side,

$$
\begin{aligned}
& A^{*}\left(x, \theta_{a}^{*}\right)-\hat{A}\left(x, \theta_{a}\right)=\psi_{a}\left(\theta_{a}^{*}-\theta_{a}\right)=-\psi_{a} \tilde{\theta}_{a} \\
& B^{*}\left(x, \theta_{b}^{*}\right)-\hat{B}\left(x, \theta_{b}\right)=\psi_{b}\left(\theta_{b}^{*}-\theta_{b}\right)=-\psi_{b} \tilde{\theta}_{b} \\
& \dot{s}=\alpha\left[\begin{array}{l}
\left(\varepsilon_{a}(x, t)-\psi_{a} \tilde{\theta}_{a}\right)+\left(\varepsilon_{b}(x, t)-\psi_{b} \tilde{\theta}_{b}\right) u_{e q}(t) \\
-B(x, t) u_{c}(t)-\beta \operatorname{sgn}(\mathrm{s})
\end{array}\right]
\end{aligned}
$$

and:

$$
\begin{gathered}
\dot{V}=s \alpha\left[\begin{array}{c}
\left(\varepsilon_{a}(x, t)-\psi_{a} \tilde{\theta}_{a}\right)+\left(\varepsilon_{b}(x, t)-\psi_{b} \tilde{\theta}_{b}\right) u_{e q}(t) \\
-B(x, t) u_{c}(t)-\beta \operatorname{sgn}(\mathrm{s})
\end{array}\right]+ \\
+\tilde{\theta}_{a} \eta_{a} \alpha \psi_{a}(\mathrm{x}) \mathrm{s}+\tilde{\theta}_{b} \eta_{b} \alpha \psi_{b}(\mathrm{x}) \mathrm{su}_{e q}
\end{gathered}
$$

where $\quad \alpha>0 ; \operatorname{s.sgn}(\mathrm{s}) \geq 0 \quad$ and $\quad$ we obtain $\dot{V} \leq 0 \quad \forall x \in \Omega$.

Theorem 2: The control input $u(t)=u_{e q}(t)-u_{c}(t)$

$u_{e q}=\hat{B}^{T}\left(\mathrm{x}, \theta_{b}^{t}\right)\left[\varepsilon_{0} I_{m}+\hat{B}\left(\mathrm{x}, \theta_{b}^{t}\right) \hat{B}^{T}\left(\mathrm{x}, \theta_{b}^{t}\right)\right]^{-1}$

$\left(-\hat{A}\left(\mathrm{x}, \theta_{a}^{t}\right)+\dot{\mathrm{x}}_{d}-\beta \operatorname{sgn}(\mathrm{s})\right)$;

with

$u_{c}(t)=B^{-1} s\left(\bar{\varepsilon}_{b}\left|u_{e q}\right|+\bar{\varepsilon}_{a}\right)$

$s=\alpha e+\xi$ ensure the nonlinear system (6) stability in finite time.

Proof:

We obtain the result:

$$
\begin{aligned}
s^{T} \dot{s} & =s^{T} \alpha\left[\begin{array}{l}
\left(\varepsilon_{a}(x, t)-\psi_{a} \tilde{\theta}_{a}\right)+\left(\varepsilon_{b}(x, t)-\psi_{b} \tilde{\theta}_{b}\right) u_{e q}(t) \\
-B(x, t) u_{c}(t)-\beta \operatorname{sgn}(\mathrm{s})
\end{array}\right] \\
& \approx s^{T} \alpha\left(\begin{array}{l}
\varepsilon_{a}(x, t)+\varepsilon_{b}(x, t) u_{e q}(t) \\
-\bar{\varepsilon}_{a}-\bar{\varepsilon}_{b}\left|u_{e q}\right|-\beta \operatorname{sgn}(\mathrm{s})
\end{array}\right) \\
& \leq-s^{T} \alpha \beta \operatorname{sgn}(\mathrm{s}) \\
& \leq-\|s\| \alpha \beta \operatorname{sgn}(\mathrm{s})
\end{aligned}
$$

Therefore, $s=\alpha e+\xi \rightarrow 0$ in finite time

Remark 1. it is necessary to ensure that the time of convergence of sliding surface is finite. The fact is described based on the following example:

We consider the system as follows:

$$
\left\{\begin{array}{l}
\frac{d x}{d t}=A x+B s \\
\frac{d s}{d t}=C x+D s
\end{array}\right.
$$

where: $A \in \mathbb{R}^{n \times n}, B \in \mathbb{R}^{n \times r}, C \in \mathbb{R}^{r \times n}, D \in \mathbb{R}^{r \times r}, s$ is the sliding surface. Selecting $A$ is Hurwitz matrix and $\left[\begin{array}{ll}A & B \\ C & D\end{array}\right]$ is not Hurwitz matrix. We obtain that although $s$ converges to 0 in infinite time, $x$ does not converge to 0 .

Remark 2. The proposed control law ensure that the system trajectory converge to sliding surface in finite time.

Remark 3. We almost utilized theorem 1 to design adaptive sliding mode control technique for multi motor systems. 
In order to eliminate the effect of chattering phenomenon, fuzzy technique (by Tagaki - Sugeno - Kang) would be proposed to adjust the coefficient $\alpha$ depending on the sliding surfaces s and $\dot{s}$, table 2, 3 and figure 3:

Table 2. Rule Matrix of control.

\begin{tabular}{|c|c|c|c|c|}
\hline \multicolumn{2}{|c|}{} & \multicolumn{3}{|c|}{$\mathrm{s}$} \\
\cline { 3 - 6 } & $\mathrm{N}$ & $\mathrm{Z}$ & $\mathrm{P}$ \\
\hline \multirow{3}{*}{$s$} & $\mathrm{~N}$ & $\mathrm{~B}$ & $\mathrm{M}$ & $\mathrm{B}$ \\
\cline { 2 - 5 } & $\mathrm{Z}$ & $\mathrm{B}$ & $\mathrm{S}$ & $\mathrm{B}$ \\
\cline { 2 - 5 } & $\mathrm{P}$ & $\mathrm{B}$ & $\mathrm{M}$ & $\mathrm{B}$ \\
\hline
\end{tabular}

Table 3. Properties of controller.

\begin{tabular}{|c|c|}
\hline AND method & MIN \\
\hline OR method & MAX \\
\hline Implication & MIN \\
\hline Aggregation & MAX \\
\hline Defuzification & Weighted average \\
\hline
\end{tabular}
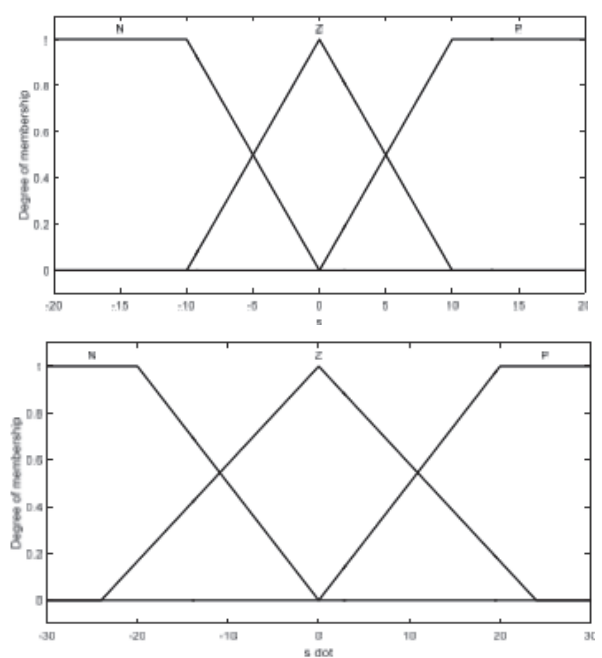

Fig. 3. Fuzzification.

\section{Simulation results}

In this section, we consider several simulation results to demonstrate the effectiveness of the proposed sliding mode control law based on the two-motor system having parameters as follows:

$n_{p 1}=n_{p 2}=4, J_{1}=J_{2}=500 \mathrm{Kgm}^{2}, L_{r 1}=0.2 \mathrm{H}$,

$L_{r 2}=0.3 H, \omega_{r 1 d}=\omega_{r 2 d}=700 \mathrm{v} / p, F_{d}=250 \mathrm{~N}$.

Figures 3, 4 show the tracking performance behaviour of velocity based on fuzzy adaptive sliding mode control law in presence of disturbance (figures 4, 5, 8). Figures 6, 7 show the high tracking performance behaviour of velocity based on adaptive sliding mode control law without disturbance.

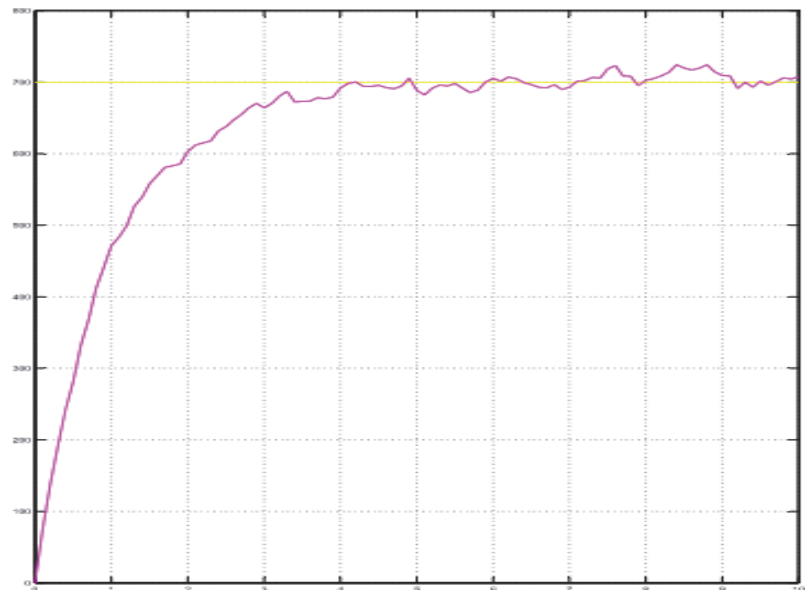

Fig. 4. The behaviour of the first motor's speed in presence of disturbance.

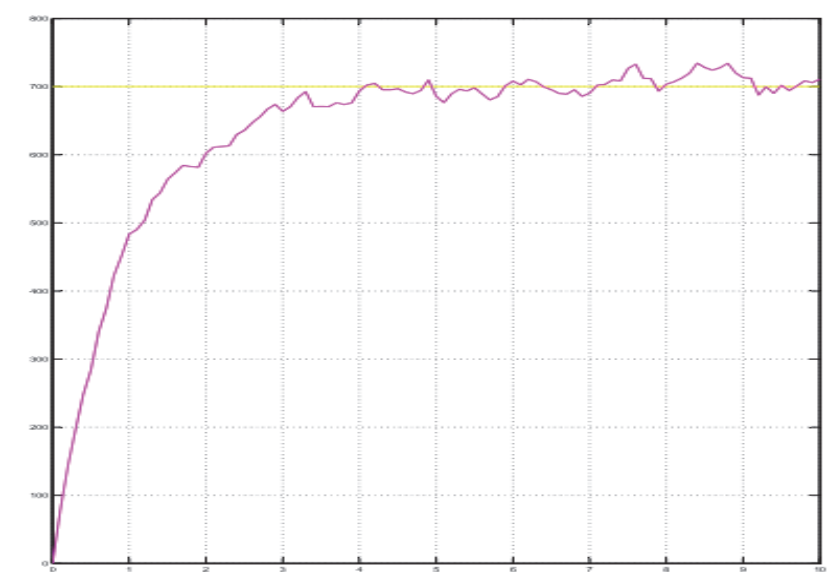

Fig. 5. The behaviour of the second motor's speed in presence of disturbance.

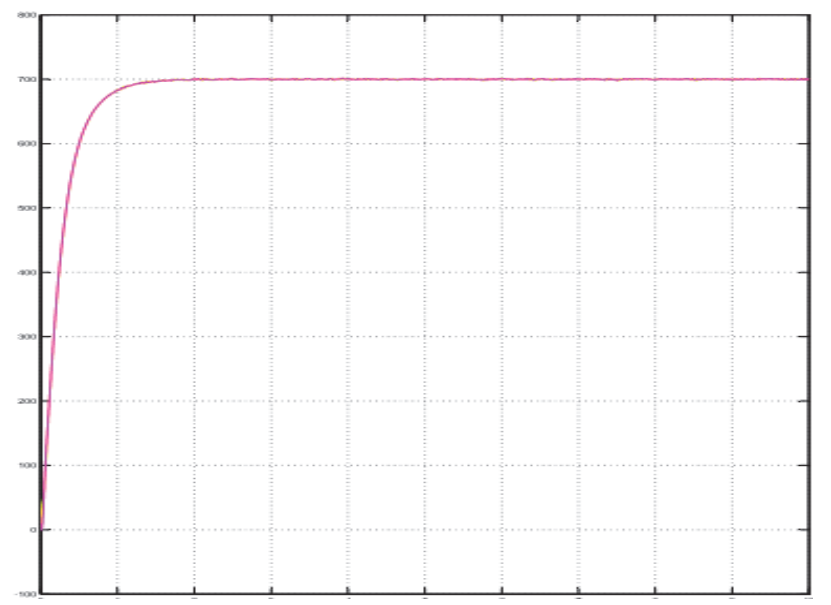

Fig. 6. The behaviour of the first motor's speed without disturbance. 


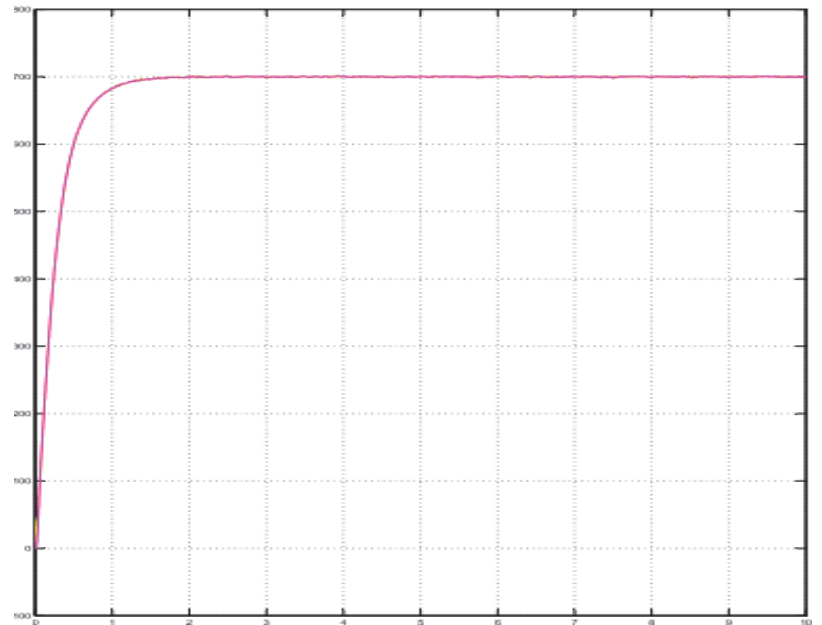

Fig. 7. The behaviour of the second motor's speed without disturbance.

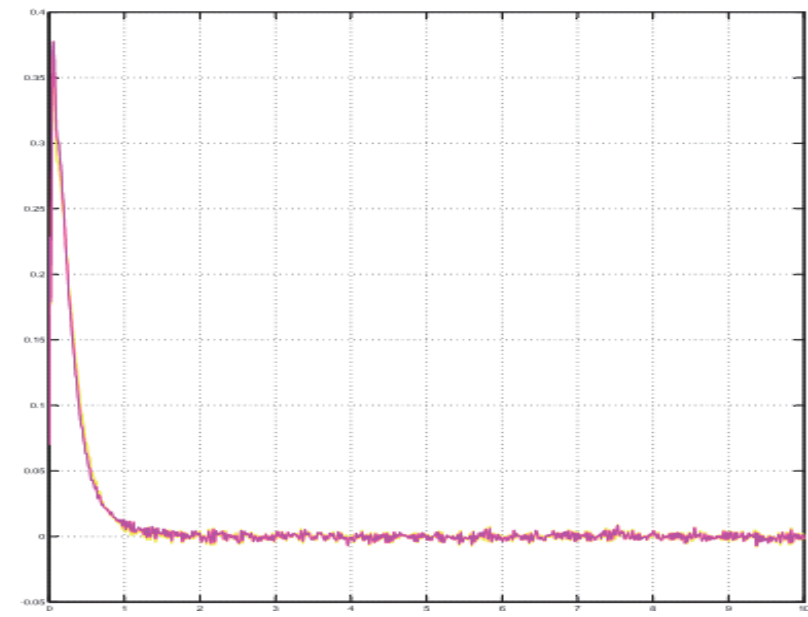

Fig. 8. The speed error between 2 motors.

\section{Conclusion}

This paper described a fuzzy adaptive sliding mode control law the two-motor system in presence of elastic and backlash, friction. The effectiveness of the proposed control scheme was pointed out by theoretical analysis and simulation results.

\section{References}

1. Y Mi et al., Proc. The 2013 International Conference on Electrical Machines and Systems, 2282-2285 (2013)

2. J Zhang et al, Proc. The IEEE International Conference on Intelligent Computing and Intelligent Systems, 178182 (2009)

3. G Liu et al., Proc. The IEEE International Conference on Networking, Sensing and Control, 1476-1479 (2008)

4. L Jinmei et al, Proc. The IEEE International Conference on Industrial Technology, 1-6 (2008)

5. N.T.T. Vu, D. Yu, H.H. Choi, J.-W. Jung, IEEE Trans. Industrial Electronics, 60 (10), 4281-4291, (2013)
6. M. Zhihong, A.P. Paplinski, H.R. Wu, IEEE Trans. Autom. Control, 39, 2464-2469 (1994)

7. J.J.E. Slotine, W. Li, Applied Nonlinear Control (Prentice Hall, New Jersey, 1991)

8. S. Labiod, M.S. Boucherit, T.M. Guerra, Fuzzy Sets Syst., 151, 59-77 (2005)

9. V. Nekoukar, A. Erfanian, Fuzzy Sets Syst., 179, 34-49 (2011) 